\title{
Comments for Current Interpretation EEG Alpha Activity: A Review and Analysis
}

\author{
Olga Bazanova \\ Biofeedback Software Laboratory, State Research Institute of Molecular Biology and Biophysics, Siberian Branch, \\ Russian Academy of Medical Sciences, Novosibirsk, Russia \\ Email: Bazanova@soramn.ru
}

Received July 25, 2011; revised November 3, 2011; accepted November 21, 2011

\begin{abstract}
Introduction: Exploring the EEG alpha oscillations generates considerable interest because there is well known its role in cognitive and psycho emotional aspects of human life. However, till now there isn't well determined definition what is alpha activity phenomena and which indices are characterize it. Method: This article focuses on the attempt to determine EEG alpha-activity phenomena, its physical, molecular and morphological nature, to highlight its indices and their role in optimal functioning. Results: Specifically, this article examines individual alpha activity indices: 1) the individual alpha peak frequency; 2) activation magnitude measured by estimation the amount of alpha amplitude suppression in response to eyes open and individual alpha band width; 3) three alpha "autorhythmicity" indices: intra-spindle amplitude variability, spindle length and steepness. Conclusions: Throughout, the article provides a number of suggestions which alpha activity indices and in which conditions could be applied in psycho physiological investigations, what is their role in optimal functioning and what are possible directions for future research.
\end{abstract}

Keywords: EEG Alpha Activity; Individual Alpha Peak Frequency; Individual Alpha Band Width; Activation; Alpha Spindle Microstructure

\section{Introduction}

Individuality is one of the most frequently investigated fields in cognition and, despite changes in research paradigms, it has not lost its relevance. From the time of the Great Russian physiologist Ivan Michailovich Sechenov, it was pointed out that the body is an amazing system with many complexities and a special potential for improving itself or feedback connections [1]. Later it became clear that such condition in healthy subjects is called "peak performance" [2,3], or "optimal functioning" [4]. The recent identification of specific brain signatures involved in optimal cognitive and psychomotor functioning demonstrate its association with general enhancement EEG alpha amplitude [5-8]. So the general line of the most prevailing argumentation $[9,10]$ have to be that alpha activity reflects a certain type of self-control (top-down) process. Despite the key role of EEG alpha activity in optimal functioning known since Berger's time, till now it is not clear which quantities characterize "alpha status:" increasing or decreasing alpha amplitude, left or right shift of spectral peak frequency, synchronization or desynchronization. Moreover the potential performance enhancing benefits of EEG alpha biofeedback remain unclear because a number of methodological factors that may impact the effectiveness of such training are unexplored. So the aims of this review are 1) to determine what phenomena could be called "EEG Alpha activity" and what brain mechanisms are underlying this, 2) which EEG parameters characterize EEG alpha activity and what is their intra- and inter individual variability and 3) what alpha-biofeedback applications benefit in optimal functioning.

From physical point of view waves activity could be measured in terms of frequency, amplitude, and phase. So it's possible to define 3 main groups of the alpha activity indices: 1) the frequency of the dominant posterior EEG rhythm [11,12], which is assessed as individual alpha peak frequency in an eyes-closed condition (IAPF) [13-15]; 2) the amount of alpha amplitude suppression in response to eyes-open $[12,16,17]$ which defines the activation magnitude, and the width of the frequency range in which amplitude suppression occurs, or alpha band width $[7,18]$; 3) the "autorhythmicity" (or bursting/spindle forming process), which is result of phase resetting process [19-21] (Figure 1).

\section{The Individual Alpha Peak Frequency (IAPF)}

Many experimental studies present evidences that IAPF varies intra-individually as a function of age: increases 
through childhood till pubertal age $[22,23]$ and decreases after 40 years of age [24]. Few investigations showed the hormonal influence on IAPF-it increases with progesterone activity enhancement during women menstrual cycle [25-28] and in conditions when cortisol blood level increases [29]. Beside it IAPF varies in dependence on resting condition and the personal cognitive involvement in a task performance $[8,10,30]$. Good performance increases IAPF, but a decrease in IAPF is always related to a drop in performance [31] and fatigue [30]. Moreover neurofeedback training (NFT) directed on increasing IAPF may be used for improvement cognitive performance [13]. From the other side our recent study of the upper alpha stimulating NFT impact on the optimal musical performance achievement demonstrated increasing IAPF only in students with baseline resting alpha frequency lower 10 $\mathrm{Hz}$ (LAF subjects). As far as students with IAPF more 10 $\mathrm{Hz}$ (HAF) their IAPF did not changed or even decreased in post-NFT resting condition [32]. So it could be proposed that different alpha peaks frequency reflect different mechanisms of brain activation and alpha waves generation. Really, overall EEG power spectrum frequency Principal Component Analysis (fPCA) yields multiple alpha factors with identical peak frequencies, which relate to different neuronal functions [33]. Gavrish \& Malykh (1994) twin study proposed that nature of the different alpha-frequency components is differently heritable. The multiple psychogenetic studying confirm the high heritability of the resting IAPF $[1,34]$.

Evidence from animal models supports that alpha wave frequency is a result of the tuning of the local cortical network, which depends on $\mathrm{Ca}^{2+} \mathrm{T}$-channels activity [3537]. Luthi \& McCormick, (1998) argued that block of the $\mathrm{Ca}^{2+}$ oscillations is associated with a block of the spindle wave refractory period such that continuous $6-$ to $10-\mathrm{Hz}$ oscillations were generated throughout the net-work.

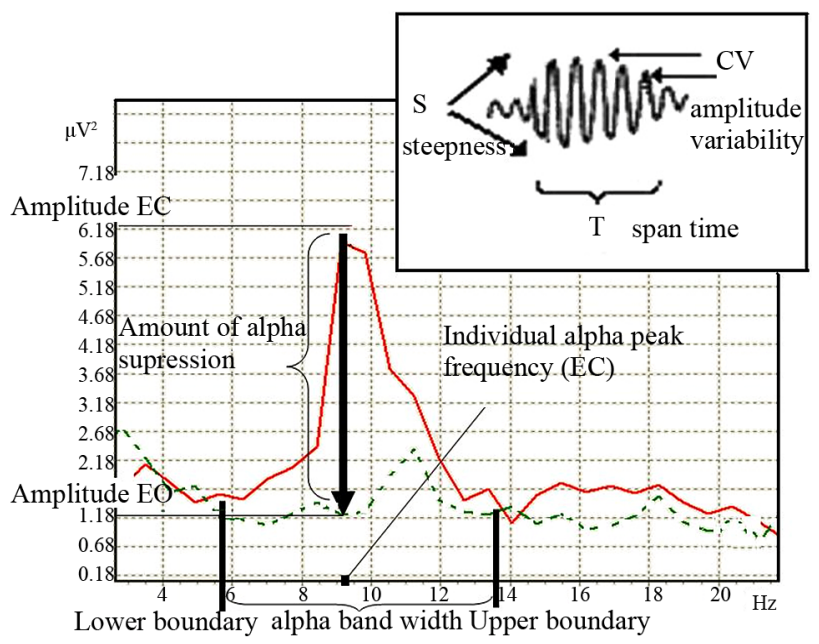

Figure 1. Alpha activity indices (EC-eyes closed-bold line spectra, EO-eyes open-dotted line spectra).
So the $\mathrm{Ca}^{2+}$ oscillations frequency determines the dominant brain frequency [38]. These experimental results revile the Michael Nikolaevich Livanov's and John Eccles hypothesis about inhibitory role of delaying the rhythmic waves generated in thalamus for brain self control and mind [1,39]. Among different alpha frequency measurements (individual alpha peak frequency, mean and center gravity) IAPF is believed to reflect aggregate alpha generation $[15,35]$. It is evident now that IAPF reflects individual genetic influences on the underlying neural mechanisms of the generation of alpha activity $[35,37]$. But the definition of appropriate frequency bands and choice of recording reference limit the interpretability of quantitative EEG, which may be further compromised by distorted topographies or inverted hemispheric asymmetries when employing conventional (non-linear) power spectra. The analysis of fixed frequency band could therefore blur the real alpha peak, masking the age-or functions related modifications. For identification experimental conditions which are the most opportunely used for learning alpha frequency endophenotypic qualities we questioned when and where topographically IAPF is the most stabile and reproducible? For it we had provided the "test-retest" EEG recordings in 96 male subjects aged 26 - 40 twice over 14 - 15 days in resting eyes closed and eyes open conditions and analyzed EEG in fixed standard $8-12 \mathrm{~Hz}$ and individually determined alpha band. It's appeared that intra-individual correlation coefficient (ICC) is the strongest in posterior brain area, in eyes closed condition and assignable in individual alpha band; the weakest - in anterior area in the eyes open condition and defined in fixed standard range [14]. We didn't find the laterality influence on the IAPF mean. Moreover the same results that genotype-dependent difference in IAPF was independent of EEG location received by Bodenmann and coauthors [40]. These data reflect the universal corticothalamic origin for alpha generation in healthy men.

Artur Nikolaevich Lebedev (1994) has proposed a functional role for the human alpha rhythm in stating that "cyclical oscillations in an alpha rhythm determine the capacity and speed of working memory. The higher frequency the greater the capacity and the speed of memory" [41,42]. In addition, Klimesch (1997) has argued that thalamo-cortical feedback loops oscillating within the alpha frequency range allow searching and identification of encoded information. He speculated that faster oscillating feedback loops would correspond to faster access to encoded information. These theories are supported by the experimental results of Klimesch, Schimke and Pfurtscheller research (1993). They argued that alpha frequency of good working memory performers lies about $1 \mathrm{~Hz}$ higher than that of poor working memory performers. We had shown that score for musical sight reading is higher in musicians with higher, than lower IAPF [32]. 
The same results were received by the functional Val158Met polymorphism of COMT study, where LAF-Val/VAL homozygote subjects demonstrated lower score in counting task than HAF-Met/MET homozygote young men [40]. So, rise the question: are smarter brains running faster? Posthuma, Neale, Boomsma and de Geus (2001) concluded that both peak frequency and the dimensions of IQ were highly heritable (range, $66 \%$ to $83 \%$ ). But large part of the genetic variance in alpha peak frequency as well as in working memory and processing speed was due to nonadditive factors. Moreover there was no evidence of a genetic correlation between alpha peak frequency and any of the four WAIS dimensions. So there must be additional EEG indexes predicting cognitive ability.

In our experiment using the median of posterior IAPF in resting eyes closed condition, 96 healthy male subjects were divided into groups; with low (LAF - IAPF $<10 \mathrm{~Hz}$ ) and high (HAF - IAPF $\geq 10 \mathrm{~Hz}$ ). alpha frequency subjects. It appeared that LAF and HAF subjects differed in psychometric strategies for achieving success in nonverbal creative tasks performance. LAF subjects emphasize originality while HAF-fluency in reaching the same score of Torrance test performance [7]. The subjects with highest and lowest IAPF level showed the highest originality score in nonverbal creativity task performance (Figure 2).

So we proposed that different behavior strategies observed in LAF and HAF subjects could be due to 1) genetic factors for individual patterns of spontaneous waking EEG and 2) different neurophysiological mechanisms of brain activation in low and high alpha frequency ranges.

\section{The Activation Magnitude}

Vaez Mousavi, Barry, Rushby and Clarke (2007) had conceptualized separately two aspects of energetic state,

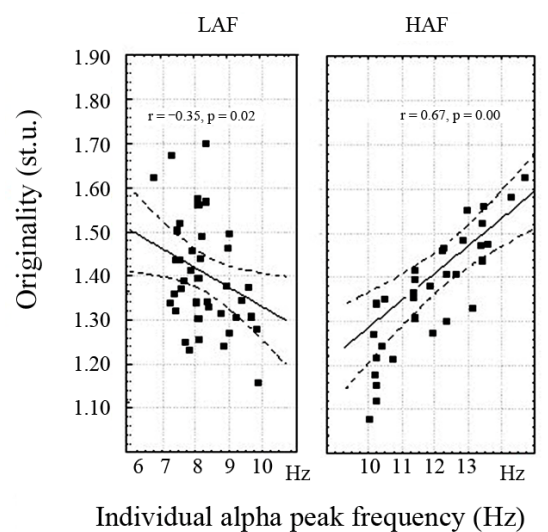

Figure 2. The interrelationships between individual alpha peak frequency and originality in nonverbal task performance in 48 low (LAF) and 48 high (HAF) alpha frequency male subjects [14]. "arousal" and "activation". "Arousal" has been defined as the energetic state at any particular time, and task-related "activation" as the task-related change in state from resting baseline to the task situation. According EEG data activation magnitude indicates the amount of alpha amplitude suppression in response to eyes-open conditions $[16,43]$. This child study confirms the generality of eyesclosed vs eyes-open EEG measures differing in mean power levels and topography, the latter indicating brain activity related to visual processing. Skin conductivity level was negatively correlated with mean alpha power levels in the eyes-closed condition, and increased significantly from eyes-closed to eyes-open. Reductions were found in across-scalp mean alpha power from eyes-closed to eyes-open. It's important to note no topographic changes were evident in real alpha band. This fact should be considered when evaluating EEG research, and in choosing baseline conditions for different paradigms [16]. These differences should be recognized when evaluating EEG research, and considered when choosing eyes-open or eyes-closed baseline conditions for different paradigms [44]. Even at 1970 Nowlis and Kamiya were able to show significant differences in alpha biofeedback impact on activation magnitude between alpha enhancement in eyes closed and eyes open trials [45].

It's known that physiological responses to stimuli in a task are dependent on the arousal level at the time of stimulus presentation, rather than the task-related activation. In contrast, performance on the task is dependent on the task-related activation, rather than the current arousal level. Doppelmayr, Klimesch, Hodlmoser, Sauseng, and Gruber (2005) found a significant interaction of task difficulty and activation in the upper alpha band, indicating both, a weaker activation for the high intelligent subjects during the easy tasks, and a significant increase from easy to difficult tasks for this group only [46]. So it could be proposed that activation magnitude varies in dependent on cognitive task difficulties. Increasing of the taskrelated relative activation magnitude varies on the intraindividuall level and particularly associates with reaction time [47] and psychomotor task performance skill [48]. Amount of alpha suppression in response to eyes open increases with maturation and decreases after 40 years old [23], depends on the neurohumoral status [27, 49] and even eyes movement direction [50]. Decreased amount of alpha suppression in response to eyes open was demonstrated in spinal cord injured in comparison with healthy able-bodied participants [51], and in attention deficit patients [52]. Moreover increased activation magnitude is result of upper alpha stimulating neuronfeedback training [32,53].

From the other side recent investigations Loo and Smalley (2008) showed that EEG measures of visual and cognitive activation are a promising avenue of study in 
the search for putative endophenotypes for ADHD [54] or for individual cognitive strategy type [54,55]. Our results of multiple regression discriminated different predictors of the amount of alpha suppression: in low alpha frequency group (LAF), - amplitude variability, but in high alpha frequency group (HAF) — alpha spindle amplitude variability [56]. Analogical different correlations of amplitude suppression (desynchronization) with EEG components in different alpha frequency peaks was obtained by Pfurtscheller and Lopes da Silva (1999). They hypothesized that with an increasing number of interconnecting neurons and there with an increasing number of coherently activated neurons, the amplitude increases and the frequency decreases. The lower alpha component in Figure $\mathbf{3}$ has a larger spectral peak magnitude than the higher alpha component [57].

Mazaheri and Jensen found too that $10 \mathrm{~Hz}$ oscillations after visual stimuli preserve their phase relationship with respect to before the stimuli, while $8 \mathrm{~Hz}$ phase-resetting oscillations are responsible for visually evoked responses [11]. These different interrelations between amount of alpha amplitude suppression (desynchronization) and other EEG indices in low and high frequency ranges is consistent with the idea that alpha desynchronization serves an integrative role through a corticocortical "gating" $[17,33]$. This means that the neurophysiological mechanisms by which activation is generated in LAF and HAF subjects are different (Bazanova et al., 2008) what was theoretically supported in previous investigations $[17,58]$.

In EEG-fMRI coupled investigations, Laufs and coauthors showed that spontaneous reductions in alpha amplitude associated with increasing cognitive activity is connected to general brain activation in a wide (not only standard alpha $8-12 \mathrm{~Hz}$ ) spectral frequency range [59]. Actually, till now, the alpha frequency range boundaries were defined on the basis of general agreement, without

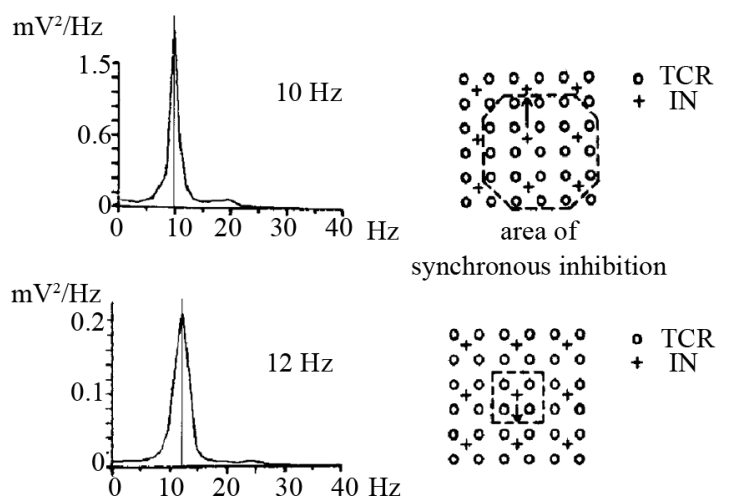

Figure 3. Results of a simulation study displaying the relationship between frequency and interconnectivity of neurons. The area of synchronous inhibition is marked. TCR thalamic relay cells; IN interneurons, (Figure from G. Pfurtscheller, \& F. H. Lopes da Silva (1999)). a theoretical basis, without respect to functional features -reactivity to visual stimulation $[21,60,61]$. Meanwhile the individual alpha bandwidth (IABW) is dependent on the neurohumoral status-in high estrogen and progesterone stages alpha band is wider than in lower hormonal phases during the female menstrual cycle [27] and gender (women have a higher low alpha band boundary frequency than men $[27,62])$. Moreover, individual alpha frequency range could vary in accordance with brain activation [7,61] (Figure 4).

For example, Individual alpha band width is wider in highly-skilled professional musicians than in non-musicians [6], and positively correlated with the overall Torrance creativity coefficient and flexibility in creative task performance [7], academic achievements [18] and biofeedback training efficiency [48].

By the results of D. Kaiser (2001) and our study it have demonstrated the importance of accounting for individual alpha ban range $[61,63]$. Neurofeedback training applied in individual EEG frequency ranges was much more efficient than neurofeedback training of standard EEG frequency ranges [18] (Figure 5). Moreover, the standard theta/beta decreasing neurofeedback training protocol could aggravate clinical condition of ADHD patient [61, 63].

The enlargement of the individual alpha bandwidth is one of the main indicators of success in both neurofeedback and heart rate decreasing biofeedback training [56]. Thus, it may be concluded that the ability for enhancing self-control could be associated with the individual alpha band broadening.
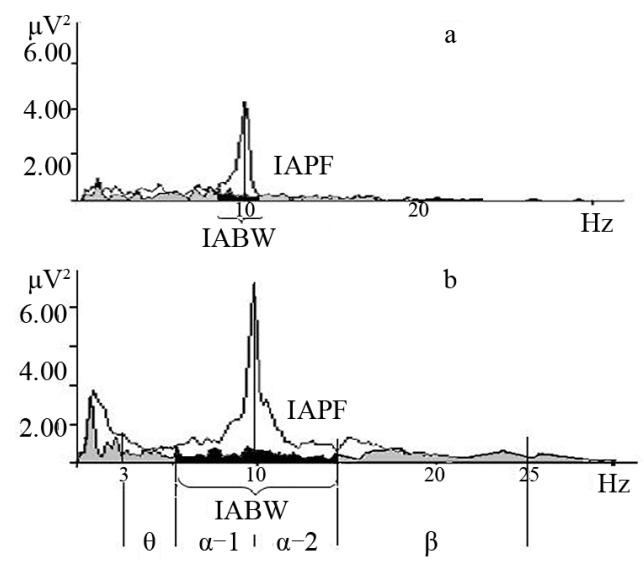

Figure 4. EEG spectral power in the parietal-occipital areas in the eyes-closed (white) and-open (gray) conditions in healthy subjects. Spectral power decreases in eyes open by more than $20 \%$ from baseline eyes closed within individual alpha ranges (black); $a$ and $b$ examples of different alpha-bandwidths with the same individual alpha peak frequency. The abscissa shows frequency, $\mathrm{Hz}$; the ordinate shows spectral power at the frequencies indicated, $\mu \mathrm{V}^{2}$; IABW is individual alpha bandwidth; IAPF is individual alpha peak frequency [18]. 

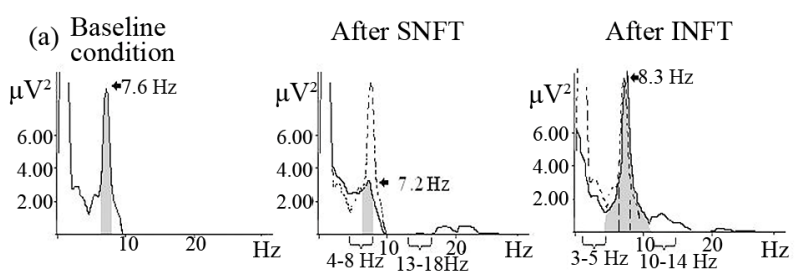

(b)

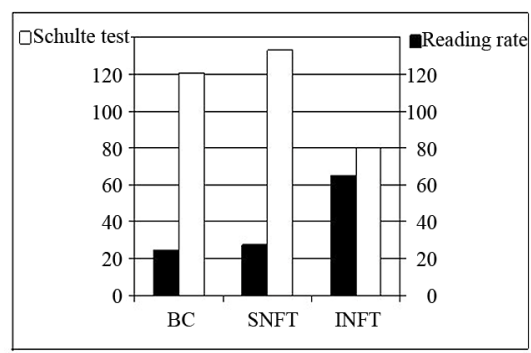

Figure 5. Spectra power (a) and psychometrical test performance (b) of boy with attention deficit disorder in baseline condition (b, c), after 10 sessions theta/beta decreasing neurofeedback training in standard EEG ranges (SNFT-theta $=4$ - $8 \mathrm{~Hz}$ and beta $=13-18 \mathrm{~Hz}$ ) and in individual EEG ranges (INFT-theta $=3-5 \mathrm{~Hz}$, beta $=10-14 \mathrm{~Hz}$ ). Grey areas denote spectral power in individual alpha band. Spectra power in baseline condition-dotted line. Numbers with arrows-individual alpha peak frequency [63].

Alpha power and activation magnitude measured as amount of alpha amplitude suppression and band width rises many questions which impossible to answer with simple spectral analysis. To overcome the limitations of conventional spectral analysis based on averaging procedures and to reveal dynamic and temporal characteristics of alpha activity, an entire set of individual shortterm stationary EEG segments may be obtained $[64,65]$. Non-stationary phenomena are present in the EEG, usually in the form of transient events, such as alternation of relatively homogenous intervals (bursting segments) with different statistical features (e.g., with different amplitude or variance) [35]. The idea that alpha oscillations have a spindle-like form only during sleep [11] has been contradicted by P. Kellaway, who described the so-called lambda waves $(8-13 \mathrm{~Hz})$, which may be identified using simple procedures [66]. The lambda wave is believed to represent occipital lobe activity in a person actively reading or scanning a room [67]. Often, the subjects have their eyes open and are looking carefully at the ceiling tiles. The technician will have the patient reproduce the activities that they felt caused the waves to appear in the first place [66]. It was proposed that the physiological basis of sleep spindles is probably very similar to lambda and alpha waves. To determine if the brain activation state would modulate the composition of alpha spatial microstates (spindles), J. L. Cantero and colleagues used spatial segmentation methods to show that 1) the mean duration of alpha spindles is longer in relaxed wakeful- ness than in drowsy periods and REM sleep and 2) the number of different amplitude values are more abundant in drowsiness than in other brain states [68].

Thus, overall, brain activation mechanisms could become clearer if we consider the third important EEG alpha activity feature - spindle-ability of alpha oscillation.

\section{The Alpha Spindle-Form}

The spindle-form or autorhythmicity of alpha oscillations is a product of the dynamics of neuronal assemblies in the underlying cortical activity [69-72]. Starting from Livanov's studies, it has been shown that spindle oscillations are essential for memory formation $[41,42,73]$ and associated with short- and mid-term synaptic plasticity [37]. A probable molecular mechanism of this phenomena was proposed by Destexhe and Sejnowski (2003). They suggested that spindling may activate the protein kinase A molecular "gate", thus opening the door for gene expression and allowing long-term changes to take place following subsequent inputs [74].

Average amplitude within segment indicates the volume of the neuronal population: indeed, the more neurons recruited into an assembly through local synchronization of their activity, the higher will be the oscillation amplitude of the corresponding assembly $[35,64,70]$. The assumption that alpha amplitude reflects inhibition mayat first glance - appear contradictory to the idea that alpha plays an active role in information processing, but the idea is that inhibition is an important factor that controls the exact timing of an oscillation. Thus, inhibition helps to establish a highly selective activation pattern [10].

Average spindle lifetime represents the functional lifespan of the neuronal population or the duration of operations produced by such a population [75]. It has been shown that longer spindles indicate a more relaxed state [76]. The spindle lifetime is correlated with fluency in cognitive task performance [7], and efficiency of the single biofeedback training session [32,48]. Additionally, alpha-spindles are longer in highly-skilled musicians than in amateurs [32]. The shortest alpha segments belong to HAF subjects with the highest individual alpha peak frequencies and LAF subjects with the lowest individual alpha peak frequencies [56]. So the longest spindles belong to persons with an average, or approximately average, $10 \mathrm{~Hz}$ individual alpha peak frequency. Multiple regression analysis showed that spindle lifespan is positively related to individual alpha peak frequency in LAF subjects, but to spindle steepness in HAF subjects [56]. These data suggest the different spindle formation neuronal mechanism in LAF and HAF subjects. The membrane bistability might play an important role in different patterns of spindles displayed by thalamocortical neurons. Actually, as shown by Fuentealba and colleagues (2005), the reticular neurons display membrane bistability, as 
indicated by two discrete electrical potential modes, with differentential responsiveness to cortical inputs [77]. Additionally in vivo [37,78] and in vitro intracellular [79] studies have revealed at least two different patterns during spontaneously occurring spindles, which may be related to the actions exerted by non-bistable and bistable neurons, respectively. Indeed, non-bistable neurons fired stronger bursts with higher intra-burst frequencies, which are assumed to generate IPSPs, $\sim 7-10 \mathrm{~Hz}$. By contrast, IPSPs with lower amplitudes and higher frequency are likely to be mainly generated by single action potentials, as they occur during the depolarizing plateau in bistable cells [77]. If we assume that longer spindles of stable brain activity imply less information to process (as reflected by a higher stability of the brain generator), whereas shorter segments imply a higher number of brain microstates caused by more different steps of information processing, then it is possible to suggest that the intra-segment alpha amplitude variability could be indexing a phasic event [80]. Probably, alpha-bursts are associated with a brain microstate change (such as sleep spindles), as demonstrated by its phasic intrusion in a desynchronized background of brain activity [68]. Important to know that intra-spindle amplitude variability decreases in coma or stupor [81], but increases during cognitive loading [75] and in relation to the ability for self-control in neuronfeedback training (18 - 20 sessions) [56].

So amplitude variability, which is associated with phase resetting intensity [80], reflects engagement of cognitive control mechanisms $[41,70,82]$. Moreover, in children the phase resetting index and intra-segment amplitude variability generally increases as a function of age [21, 23]. Probably, this reflects the well-known fact that ability for self-control develops with age [83].

Therefore, alpha activity indices (individual alpha peak frequency, amount of alpha suppression and individual alpha bandwidth, segmental parameters of alpha spindle) characterize the degree of cortical ability for activation, neuronal plasticity, excitability and inhibition. In this way these parameters could characterize self-regulation ability.

\section{The Alpha Activity Phenomena Hypothesis}

According the Klimesh's "time inhibition" theory the active role of alpha waves is seen in a mechanism that may also underlie the functional role of other oscillations [10]. Synchronization in the alpha frequency range helps neurons in distributed networks to effectively activate common target cells $[9,10]$. This alpha-frequency dependent mechanism plays an important role in the top-down control of cortical activation. Mechanisms giving rise to alpha amplitude desynchronization also reflect the dependence of brain activation on the alpha frequency range.
Klimesh has proposed that upper-frequency alpha oscillations are related to top-down processes in a complex sensory-motor system that controls the access to and manipulation of stored information [10]. For example, when a task requires that certain types of processes be performed with stored information (e.g., stored information must be kept in mind, must not be retrieved or must be manipulated such as with highly-skilled musicians during musical performance), individual alpha-activity increases ("alpha status" develops). As an overall brain activity, top-down control is not a unitary phenomenon. Recent Ben-Simon, Podlipsky, Arieli, Zhdanov and Hendler (2008) combined fMRI/EEG study proposed two parallel patterns of alpha modulations and underpin their anatomical basis in the human brain. These findings suggest that the human alpha rhythm represents at least two simultaneously occurring processes which characterize the "resting brain"; one is related to expected change in sensory information, while the other is endogenous and independent of stimulus change. Although twin studies have long shown that heritability of EEG oscillations in waking is substantial [78], very little is known about the genes underlying distinct EEG traits. Early linkage analyses identified a genetic locus on the distal part of chromosome $20 \mathrm{q}$ to modulate alpha power which authors named "alpha-activity" [84]. It is likely, however, that multiple genes contribute to "alpha phenotypes", and a few candidate genes were indeed found to affect alpha oscillations. So, recent genetic polymorphism studies indicated that the gene on chromosome 5q13-14 of corticotrophin releasing hormone-binding protein (CRH-BP) modulates alpha power in isolated Plains American Indians and Caucasians [85]. Moreover, a functional variation in exon 7 of the gene on chromosome 6 encoding the human GABAB receptor (GABABR1) also influences EEG voltage in the alpha range [86]. Finally, more recent data of Bodenmann and coauthors (2009) demonstrate that mechanisms involving COMT contribute to inter-individual differences in alpha oscillations frequency, which are functionally related to executive performance [40]. Thus presented here data emphasize the genetically different EEG patterns and connected with them behavioral strategies in dependence on alpha waves frequency.

\section{Conclusion}

Thus it could be concluded that studying the alpha-activity does not mean investigation only alpha amplitude intra- and inter-individual variability. EEG Alpha activity phenomena analysis should include in line with amplitude two other important physical characteristics: frequency and phase of alpha oscillations. Moreover the most interesting electrophysiological evidence that alpha oscillations play an active role in cognitive processing and self-regulation suggests that they will be different in de- 
pendence on the high or low alpha waves frequencies generated. The neuronal activation strategies for achieving the "alpha status" as a result of obvious training or biofeedback-training are different according to the individual alpha frequency too. Several factors may be common in the generation of different types of oscillations. Nonetheless, the exact mechanisms for generating an oscillation may differ widely between different frequency waves such as low and high frequency alpha, depending on individual network properties, cell types, cell physicology, hormone level, brain cells blood feeding etc. Role such biological factors as immunological, neurohumoral, activity of heart-vessel and breathing systems in generation and forming alpha oscillations is still unknown. Thus, for a better understanding the functional role of alpha activity indices in peak performance achievement through Biofeedback or BCI technologies, the investigation of cortico-visceral interplay in dependence on the inherited alpha frequency EEG types may be of crucial importance.

\section{Acknowledgements}

This research was supported by Russian Humaniterian Science Foundation Grants 10-06-00265a and BIAL Grant 45/08. I thank Prof. A. N. Lebedev, Prof. M. B. Shtark, and Prof. L. I. Aftanas for discussion and comments on this manuscript.

\section{REFERENCES}

[1] J. C. Eccles, "How the Self Controls Its Brain," SpringerVerlag, Berlin, 1994. doi:10.1007/978-3-642-49224-2

[2] R. N. Singer, "Preperformance State, Routines, and Automaticity: What Does It Take to Realize Expertise in SelfPaced Events?" Journal of Sport \& Exercise Psychology, Vol. 24, 2002, pp. 359-375.

[3] D. Vernon, "Human potential: Exploring Techniques Used to Enhance Human Performance," Routledge, London, 2009.

[4] T. Keefe, "Optimal Functioning: The Eastern Ideal in Psychotherapy," Journal of Contemporary Psychotherapy, Vol. 10, No. 1, 1978, pp. 16-24. doi:10.1007/BF01815860

[5] J. Baumeister, K. Reinecke, H. Liesen and M. Weiss, "Cortical Activity of Skilled Performance in a Complex Sports Related Motor Task," European Journal of Applied Physiology, Vol. 104, No. 4, 2008, pp. 625-631. doi:10.1007/s00421-008-0811-x

[6] O. M. Bazanova, A. V. Gvozdev, F. A. Mursin, E. G. Verevkin and M. B. Shtark, "EEG-EMG Dimensionality of the Musical Performance," Cognitive Processing, Vol. 4, 2003, pp. 33-47.

[7] O. M. Bazanova and L. I. Aftanas, "Individual Measures of Electroencephalogram Alpha Activity and Non-Verbal Creativity," Neuroscience and Behavavioral Physiology, Vol. 38, No. 3, 2008, pp. 227-235. doi:10.1007/s11055-008-0034-y
[8] F. Hummel, R. Saur, S. Lasogga, C. Plewnia, M. Erb, D. Wildgruber, W. Grodd and C. Gerloff, "To Act or Not to Act: Neural Correlates of Executive Control of Learned Motor Behavior," NeuroImage, Vol. 23, No. 4, 2004, pp. 1391-1401. doi:10.1016/j.neuroimage.2004.07.070

[9] E. Basar, "The Theory of the Whole-Brain-Work," International Journal of Psychophysiology, Vol. 60, No. 2, 2006, pp. 133-138. doi:10.1016/j.ijpsycho.2005.12.007

[10] W. Klimesch, P. Sauseng and S. Hanslmayr, "EEG Alpha Oscillations: The Inhibition-Timing Hypothesis," Brain Research Reviews, Vol. 53, No. 1, 2007, pp. 63-88. doi:10.1016/j.brainresrev.2006.06.003

[11] E. Niedermeyer, "The Normal EEG of the Waking Adult," In: E. Niedermeyer and F. Lopes da Silva, Eds. Electroencephalography: Basic Principles, Clinical Applications and Related Fields, 4th Edition, Williams and Wilkins, Philadelphia, 1999, pp. 149-173.

[12] P. Nunez, B. Wingeier and R. Silberstein, "Spatial-Temporal Structures of Human Alpha Rhythms: Theory, Microcurrent Sources, Multiscale Measurements, and Global Binding of Networks," Human Brain Mapping, Vol. 13, No. 3, 2001, pp. 125-164. doi:10.1002/hbm.1030

[13] E. Angelakis, S. Stathopoulou and J. L. Frymiare, "EEG Neurofeedback: A Brief Overview and an Example of Peak Alpha Frequency Training for Cognitive Enhancement in the Elderly," Clinical Neuropsychologist, Vol. 21, No. 1, 2007, pp. 110-129. doi:10.1080/13854040600744839

[14] O. M. Bazanova, "Individual Alpha Peak Frequency Variability and Reproducibility in Various Experimental Conditions," Zh Vyssh Nerv Deiat im IP Pavlova, Vol. 61, No. 1, 2011, pp. 102-111.

[15] G. S. Hooper, "Comparison of the Distributions of Classical and Adaptively Aligned EEG Power Spectra," International Journal of Psychophysiology, Vol. 55, No. 2, 2005, pp. 179-189. doi:10.1016/j.ijpsycho.2004.07.008

[16] R. J. Barry, A. R. Clarke, S. J. Johnstone and C. R. Brown, "EEG Differences in Children between Eyes-Closed and Eyes-Open Resting Conditions," Clinical Neurophysiology, Vol. 120, No. 10, 2009, pp. 1806-1811. doi:10.1016/j.clinph.2009.08.006

[17] K. Kirschfeld, "The Physical Basis of Alpha Waves in the Electroencephalogram and the Origin of the 'Berger Effect'," Biological Cybernetics, Vol. 92, No. 3, 2005, pp. 177-185. doi:10.1007/s00422-005-0547-1

[18] O. M. Bazanova and L. I. Aftanas, "Relationships between Learnability and Individual Indices of EEG Alpha Activity," Annals of General Psychiatry, Vol. 5, Suppl. 1, 2006, pp. 74-75. doi:10.1186/1744-859X-5-S1-S74

[19] A. M. Ivanitskiy and A. N. Lebedev, "Solving the Riddle of the Brain Rhythms," Zh Vyssh Nerv Deiat im IP Pavlova, Vol. 57, No. 5, 2007, pp. 636-640.

[20] I. Timofeev, F. Grenier, M. Bazhenov, A. R. Houweling, T. J. Sejnowski and M. T. Steriade, "Short- and MediumTerm Plasticity Associated with Augmenting Responses in Cortical Slabs and Spindles in Intact Cortex of Cats in Vivo," Journal of Physiology, Vol. 542, 2002, pp. 583598. doi:10.1113/jphysiol.2001.013479 
[21] R. W. Thatcher, D. M. North and C. J. Biver, "Intelligence and EEG Phase Reset: A Two Compartmental Model of Phase Shift and Lock," Neuroimage, Vol. 42, No. 4, 2008, pp. 1639-1653. doi:10.1016/j.neuroimage.2008.06.009

[22] T. A. Stroganova, E. V. Orekhova and I. N. Posikera, "EEG Alpha Rhythm in Infants," Clinical Neurophysiology, Vol. 110, No. 6, 1999, pp. 997-1012. doi:10.1016/S1388-2457(98)00009-1

[23] O. M. Bazanova, "Age Related Alpha Activity Change Differs for Males and Females and for Low and High Alpha Frequency EEG Pattern," Revista Espanola de Neuropsicologia, Vol. 10, 2008, pp. 82-83.

[24] R. C. Clark, D. Veltmeyer, R. J. Hamilton, E. Simms, R. Paul, D. Hermens and E. Gordon, "Spontaneous Alpha Peak Frequency Predicts Working Memory Performance Across the Age Span," International Journal of Psychophysiology, Vol. 53, No. 1, 2004, pp. 1-9. doi:10.1016/j.ijpsycho.2003.12.011

[25] F. C. Baker and I. M. Colrain, "Daytime Sleepiness, Psychomotor Performance, Waking EEG Spectra and Evoked Potentials in Women with Severe Premenstrual Syndrome," Journal of Sleep Research, Vol. 19, No. 1, 2010, pp. 214-227. doi:10.1111/j.1365-2869.2009.00782.x

[26] D. Becker, O. D. Creutzfeldt, M. Schwibbe and W. Wuttke, "Electrophysiological and Psychological Changes Induced by Steroid Hormones in Men and Women," Acta Psychiatrica Belgica, Vol. 80, No. 5, 1980, pp. 674-697.

[27] O. M. Bazanova and E. M. Mernaya, "Alpha-Activity Fluctuations in Various Hormonal States and Associated with Them Musical Performance Proved Differently in the Opposite Individual Alpha Peak Frequency Groups," Revista Espanola de Neuropsicologia, Vol. 10, No. 1, 2008, pp. 100-101.

[28] S. Solis-Ortiz, M. A. Guevara and M. Corsi-Cabrera, "Performance in a Test Demanding Prefrontal Functions Is Favored by Early Luteal Phase Progesterone: An Electroencephalographic Study," Psychoneuroendocrinology, Vol. 27, No. 8, 2004, pp. 1047-1057.

doi:10.1016/j.psyneuen.2003.10.007

[29] M. Tops, J. M. van Peer, A. E. Wester, A. A. Wijers and J. Korf, "State-Dependent Regulation of Cortical Activity by Cortisol: An EEG Study," Neuroscience Letters, Vol. 404, No. 2, 2006, pp. 39-43. doi:10.1016/j.neulet.2006.05.038

[30] S. C. Ng and P. Raveendran, "EEG Peak Alpha Frequency as an Indicator for Physical Fatigue Medicon," IFMBE Proceedings, Vol. 16, 2007, pp. 517-520. doi:10.1007/978-3-540-73044-6_132

[31] W. Klimesch, H. Schimke and G. Pfurtscheller, "Alpha Frequency, Cognitive Load and Memory Performance," Brain Topography, Vol. 5, No. 3, 1993, pp. 241-251. doi:10.1007/BF01128991

[32] O. M. Bazanova, E. M. Mernaya and M. B. Shtark, "Biofeedback in Psychomotor Training. Electrophysiological Basis," Neuroscience and Behavioral Physiology, Vol. 39, No. 5, 2009, pp. 437-454. doi:10.1007/s11055-009-9157-z

[33] C. E. Tenke and J. Kayser, "Reference-Free Quantifica- tion of EEG Spectra: Combining Current Source Density (CSD) and Frequency Principal Components Analysis (fPCA)," Clinical Neurophysiology, Vol. 116, No. 12, 2005, pp. 2826-2846. doi:10.1016/j.clinph.2005.08.007

[34] C. M. Smit, M. J. Wright, N. K. Hansell, G. M. Geffen and N. G. Martin, "Genetic Variation of Individual Alpha Frequency (IAF) and Alpha Power in a Large Adolescent Twin Sample," International Journal of Psychophysiology, Vol. 61, No. 2, 2006, pp. 235-243. doi:10.1016/i.ijpsycho.2005.10.004

[35] F. H. Lopes da Silva, "Neural Mechanisms Underlying Brain Waves: From Neural Membranes to Networks," Electroencaphalography and Clinical Neurophysiology, Vol. 79, No. 2, 1991, pp. 81-93. doi:10.1016/0013-4694(91)90044-5

[36] M. Steriade, P. Gloor, R. R. Llinas, F. H. Lopes de Silva and M. M. Mesulam, "Basic Mechanisms of Cerebral Rhythmic Activities," Electroencaphalography and Clinical Neurophysiology, Vol. 76, No. 3, 1990, pp. 481-508.

[37] M. Steriade and I. Timofeev, "Neuronal Plasticity in Thalamocortical Networks during Sleep and Waking Oscillations," Neuron, Vol. 37, No. 4, 2003, pp. 563-576. doi:10.1016/S0896-6273(03)00065-5

[38] A. Lüthi and D. A. McCormick, "H-Current: Properties of a Neuronal and Network Pacemaker," Neuron, Vol. 21, 1998, pp. 9-12.

[39] M. N. Livanov, "Rhythms of the Electroencephalogram and Their Functional Significance," Zh Vyssh Nerv Deiat im IP Pavlova, Vol. 34, 1984, pp. 613-626.

[40] S. Bodenmann, T. Rusterholz, R. Dürr, C. Stoll, V. Bachmann, E. Geissler, K. Jaggi-Schwarz and H. P. Landolt, "The Functional Val158Met Polymorphism of COMT Predicts Interindividual Differences in Brain Alpha Oscillations in Young Men," Journal of Neuroscience, Vol. 29, No. 35, 2009, pp. 10855-10862. doi:10.1523/JNEUROSCI.1427-09.2009

[41] A. N. Lebedev, "The Neurophysiological Parameters of Human Memory," Neuroscience and Behavioral Physiology, Vol. 24, No. 3, 1994, pp. 254-259. doi:10.1007/BF02362031

[42] A. N. Lebedev, "Mikhail Nikolaevich Livanov (on His 100th Anniversary of His Scientific, Scientific-Organizational, Pedagogical and Public Activities)," Usp Fiziol Nauk, Vol. 37, No. 3, 2006, pp. 87-94.

[43] M. Toscani, T. Marzi, S. Righi, M. P. Viggiano and S. Baldassi, "Alpha Waves: A Neural Signature of Visual Suppression," Experimental Brain Research, Vol. 207, No. 3-4, pp. 2010213-2010219.

[44] J. R. Cram, R. J. Kohlenberg and M. Singer, "Operant Control of Alpha EEG," Psychosomatic Medicine, Vol. 39, No. 1, 1977, pp. 11-18.

[45] D. Nowlis and J. Kamiya, "The Control of EEG Alpha Rhythm through Auditory Feedback and the Associated Mental Activity," Psychophysiology, Vol. 6, No. 4, 1970. pp. 476-484. doi:10.1111/j.1469-8986.1970.tb01756.x

[46] M. Doppelmayr, W. Klimesch, K. Hodlmoser, P. Sauseng and W. Gruber, "Intelligence Related Upper Alpha Desynchronization in a Semantic Memory Task," Brain Re- 
search Bulletin, Vol. 66, No. 2, 2005, pp. 171-177. doi:10.1016/j.brainresbull.2005.04.007

[47] S. M. Vaez Mousavi, R. J. Barry, J. A. Rushby and A. R. Clarke, "Arousal and Activation Effects on Physiological and Behavioral Responding during a Continuous Performance Task," Acta Neurobiologiae Experimentalis (Wars), Vol. 67, No. 4, 2007, pp. 461-470.

[48] O. M. Bazanova, A. Kondratenko, O. Kondratenko, E. Mernaya and E. Zhimulev, "New Computer-Based Technology to Teach Peak Performance in Musicians," Information Technology Interfaces, Vol. 7, 2007, pp. 39-44.

[49] H. Mantanus, M. Ansseau, J. J. Legros and M. TimsitBerthier, "Relationship between Dexamethasone Suppression Test and Contingent Negative Variation in Major Depressive Patients," Clinical Neurophysiology, Vol. 18, No. 4, 1988, pp. 345-353. doi:10.1016/S0987-7053(88)80091-1

[50] V. Kaiser, B. Clemens, R. Leeb, Ch. Neuper and G. Pfurtscheller, "Investigation of Cue-Based Vertical and Horizontal Eye Movements with Electroencephalographic and Eye-Tracking Data," Clinical Neurophysiology, Vol. 120, No. 11, 2009, pp. 1988-1993. doi:10.1016/j.clinph.2009.09.001

[51] R. A. Thuraisingham, Y. Tran, P. Boord and A. Craig, "Analysis of Eyes Open, Eye Closed EEG Signals Using Second-Order Difference Plot," Medical \& Biological Engineering \& Computing, Vol. 45, No. 12, 2007, pp. 12431249. doi:10.1007/s11517-007-0268-9

[52] R. J. Barry, A. R. Clarke and S. J. Johnstone, "A Review of Electrophysiology in Attention-Deficit/Hyperactivity Disorder: I. Qualitative and Quantitative Electroencephalography," Clinical Neurophysiology, Vol. 114, No. 2, 2003, pp. 171-183. doi:10.1016/S1388-2457(02)00362-0

[53] R. Shmelkina, "Some EEG Findings Caused by Real and Imaginary Stimuli in Patients and Healthy Subjects," Applied Psychophysiology and Biofeedback, Vol. 24, No. 2, 1999, pp. 143-148.

[54] S. K. Loo, S. T. Hale, G. Hanada, J. Macion, A. Shrestha, J. J. McGough, J. T. McCracken, S. Nelson and S. L. Smalley, "Familial Clustering and DRD4 Effects on Electroencephalogram Measures in Multiplex Families with Attention Deficit/Hyperactivity Disorder," Journal of American Academy Child and Adolescent Psychiatry, Vol. 49, No. 11, 2010, pp. 368-377.

[55] A. M. Ivanitsky, G. A. Ivanitsky and O. V. Sysoeva, "Brain Science: On the Way to Solving the Problem of Consciousness," International Journal of Psychophysiology, Vol. 73, No. 2, 2009, pp. 101-108. doi:10.1016/j.ijpsycho.2009.02.004

[56] O. M. Bazanova, O. A. Jafarova, E. M. Mernaya, K. B. Mazhirina and M. B. Shtark, "Optimal Functioning: Psychophysiological Bases and Neurofeedback Training," International Journal of Psychophysiology, Vol. 69, No. 3, 2008, pp. 164-165. doi:10.1016/j.ijpsycho.2008.05.422

[57] G. Pfurtscheller and T. H. Lopes da Silva, "Event-Related EEG/MEG Synchronization and Desynchronization: Basic Principles," Clinical Neurophysiology, Vol. 110, No. 11, 1999, pp. 1842-1857. doi:10.1016/S1388-2457(99)00141-8
[58] A. Mazaheri and O. Jensen, "Posterior Alpha Activity Is Not Phase-Reset by Visual Stimuli," PNAS, Vol. 103, No. 8, 2006, pp. 2948-2952. doi:10.1073/pnas.0505785103

[59] H. Laufs, J. L. Holt, R. Eltont, M. Krams, J. S. Paul, K. Krakow and A. Kleinschmidt, "Where the BOLD Signal Goes When Alpha EEG Leaves," Neuroimage, Vol. 31, No. 4, 2006, pp. 1408-1418. doi:10.1016/j.neuroimage.2006.02.002

[60] M. Arns, J. Gunkelman, M. Breteler and D. Spronk, "EEG Phenotypes Predict Treatment Outcome to Stimulants in Children with ADHD," Journal of Integrative Neuroscience, Vol. 7, No. 3, 2008, pp. 421-438. doi:10.1142/S0219635208001897

[61] D. A. Kaiser, "Rethinking Standard Bands," Journal of Neurofherapy, Vol. 5, No. 1-2, 2001, pp. 96-101. doi:10.1300/J184v05n01_08

[62] J. Carrier, S. Land, D. J. Buysse, D. J. Kupfer and T. H. Monk, "The Effects of Age and Gender on Sleep EEG Power Spectral Density in the Middle Years of Life (Ages 20 - 60 Years Old)," Psychophysiology, Vol. 38, No. 2, 2001, pp. 232-242. doi:10.1111/1469-8986.3820232

[63] O. M. Bazanova and L. I. Aftanas, "Individual EEG Alpha Activity Analysis for Enhancement Neurofeedback Efficiency: Two Case Studies," Journal of Neurotherapy, Vol. 14, No. 3, 2010, pp. 244-253. doi:10.1080/10874208.2010.501517

[64] A. Ia. Kaplan, "The Problem of the Segmental Description of the Human Electroencephalogram," Fiziol Cheloveka, Vol. 25, No. 1, 1999, pp. 125-133.

[65] D. N. Towers and J. J. Allen, "A Better Estimate of the Internal Consistency Reliability of Frontal EEG Asymmetry Scores," Psychophysiology, Vol. 46, No. 1, 2009, pp. 132-142. doi:10.1111/j.1469-8986.2008.00759.x

[66] P. Kellaway, "Orderly Approach to Visual Analysis: Elements of the Normal EEG and Their Characteristics in Children and Adults," In: J. S. Ebersole and T. A. Pedley, Eds., Current Practice of Clinical Electroencephalography, 3rd Edition, Lippincott Williams and Wilkins, Philadelphia, 2003, pp. 100-159.

[67] O. Dimigen, M. Valsecchi, W. Sommer and R. Kliegl "Human Microsaccade-Related Visual Brain Responses," Journal of Neuroscience, Vol. 29, No. 39, 2009, pp. 1232112331. doi:10.1523/JNEUROSCI.0911-09.2009

[68] J. L. Cantero, M. Atienza, R. M. Salas and C. M. Gomez, "Brain Spatial Microstates of Human Spontaneous Alpha Activity in Relaxed Wakefulness, Drowsiness Period, and REM Sleep," Brain Topography, Vol. 1, 1999, pp. 1257 1263.

[69] V. B. Dorokhov, "Alpha-Bursts and K-Complex: Phasic Activation Pattern during Spontaneous Recovery of Correct Psychomotor Performance at Difference Stages of Drowsiness," Zh Vyssh Nerv Deiat im IP Pavlova, Vol. 53, No. 4, 2003, pp. 503-512.

[70] M. N. Livanov and V. N. Dumenko, "The Neurophysiological Aspect of Research on the Systems Organization of Brain Activities," Usp Fiziol Nauk, Vol. 18, No. 3, 1987, pp. 6-16.

[71] D. Lehmann, W. K. Strik, B. Henggeller and M. Koukkou, 
"Microstates in Spontaneous Momentary EEG Potential Maps during Visual Imagery and Abstract Thought," Brain Topography, Vol. 6, 1994, p. 251.

[72] W. Singer, A. K. Engel, A. S. Kreiter, M. H. Munk, S. Neuenschwander and P. R. Roelfsema, "Neuronal Assemblies: Nessesity, Signature and Detectability," Trends in Cognitive Sciences, Vol. 1, No. 7, 1997, pp. 252-261. doi:10.1016/S1364-6613(97)01079-6

[73] S. Gais, W. Plihal, U. Wagner and J. Born, "Early Sleep Triggers Memory for Early Visual Discrimination Skills," Nature Neuroscience, Vol. 3, 2000, pp. 1335-1339. doi:10.1038/81881

[74] A. Destexhe and T. J. Sejnowski, "Interactions between Membrane Conductance Underlying Thalamocortical SlowWave Oscillations," Physiological Reviews, Vol. 83, 2003, pp. 1401-1453.

[75] A. Ia. Kaplan, S. V. Borisov, S. L. Shishkin and V. A. Ermolaev, "Analysis of the Segmental Structure of EEG Alpha-Activity in Humans," Ross Fiziol Zh im IP Sechenova, Vol. 88, No. 4, 2002, pp. 432-442.

[76] E. Huupponen, A. Maksimow, P. Lapinlampi, M. Särkelä, A. Saastamoinen, A. Snapir, H. Scheinin, M. Scheinin, P. Meriläinen, S. L. Himanen and S. Jääskeläinen, "Electroencephalogram Spindle Activity during Dexmedetomidine Sedation and Physiological Sleep," Acta Anaesthesiologica Scandinavica, Vol. 52, No. 2, 2008, pp. 289-294. doi:10.1111/j.1399-6576.2007.01537.x

[77] P. Fuentealba, I. Timofeev, M. Bazhenov, T. J. Sejnowski and M. Steriade, "Membrane Bistability in Thalamic Reticular Neurons during Spindle Oscillations," Journal of Neurophysiology, Vol. 93, No. 1, 2005, pp. 294-304. doi:10.1152/jn.00552.2004

[78] M. Steriade and R. R. Llinas, "The Functional States of the Thalamus and the Associated Neuronal Interplay," Physiological Reviews, Vol. 68, No. 3, 1988, pp. 649-742.

[79] T. Bal and D. A. McCormick, "What Stops Synchronized
Thalamocortical Oscillations?" Neuron, Vol. 17, No. 2, 1996, pp. 297-308. doi:10.1016/S0896-6273(00)80161-0

[80] S. A. Oprisan, A. A. Prinz and C. C. Canavier, "Phase Resetting and Phase Locking in Hybrid Circuits of One Model and One Biological Neuron," Biophysical Journal, Vol. 87, No. 4, 2004, pp. 2283-2298. doi:10.1529/biophysj.104.046193

[81] R. P. Brenner, "The Interpretation of the EEG in Stupor and Coma," Neurologist, Vol. 11, 2005, pp. 271-284. doi:10.1097/01.nrl.0000178756.44055.f6

[82] S. Hanslmayr, P. Sauseng, M. Doppelmayr, W. Gruber, M. Doppelmayr, R. Freunberger, T. Pecherstorfer and N. Birbaumer, "Alpha Phase Reset Contributes to the Generation of ERPs," Cerebral Cortex, Vol. 17, No. 1, 2007, pp. 1-8. doi:10.1093/cercor/bhj129

[83] W. Mischel, "Toward an Integrative Science of the Person," Annual Review of Psychology, Vol. 55, 2004, pp. 1-22. doi:10.1146/annurev.psych.55.042902.130709

[84] A. P. Anokhin, O. Steinlein, C. Fischer, Y. Mao, P. Vogt, E. Schalt and F. A. Vogel, "Genetic Study of the Human Low-Voltage Electroencephalogram," Human Genetics, Vol. 90, No. 1-2, 1992, pp. 99-112. doi:10.1007/BF00210751

[85] M. A. Enoch, P. H. Shen, F. Ducci, Q. Yuan, J. Liu, K. V. White, B. Albaugh, C. A. Hodgkinson and D. Goldman, "Common Genetic Origins for EEG, Alcoholism and Anxiety: The Role of CRH-BP," PLOS ONE, Vol. 3, No. 10, 2008, Article ID e3620.

[86] G. Winterer, R. Mahlberg, M. N. Smolka, J. Samochowiec, M. Ziller, H. P. Rommelspacher, W. M. Herrmann, L. G. Schmidt and T. Sander, "Association Analysis of Exonic Variants of the GABA(B)-Receptor Gene and Alpha Electroencephalogram Voltage in Normal Subjects and Alcohol-Dependent Patients," Behavior Genetics, Vol. 33, No. 1, 2003, pp. 7-15. doi:10.1023/A:1021043315012 